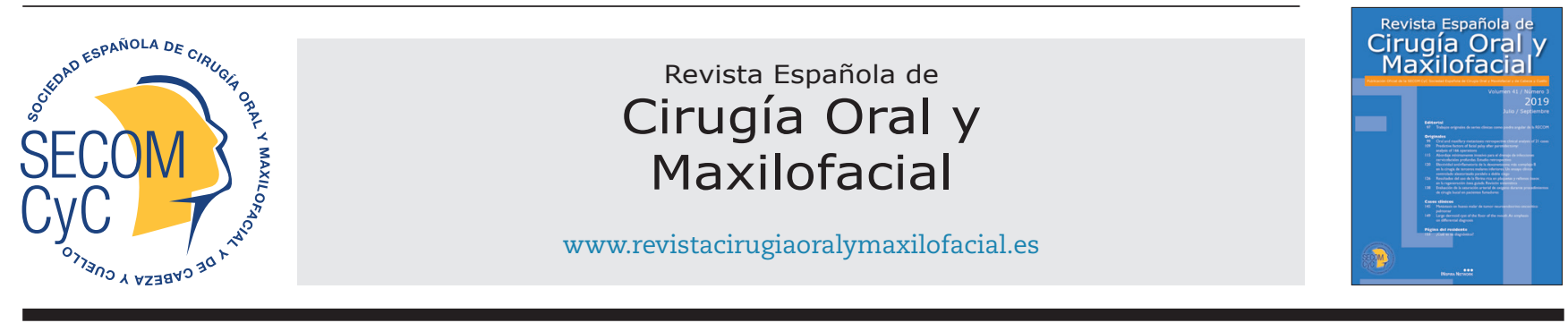

\title{
Original
}

\section{Evaluación de la saturación arterial de oxígeno durante procedimientos de cirugía bucal en pacientes fumadores}

\author{
Gisselle Fariñas Fuentes ${ }^{a}$, Zhenia Mercedes Fuentes Alcaláb, Isidoro Ortiz Arysmendysc, \\ Yavicmar Sused Beirutty Alayón $n^{d}$ y José Adolfo Cedeño Martínez ${ }^{e}$
}

${ }^{a}$ Odontóloga, Especialista en Cirugía Bucal, Universidad Central de Venezuela, Caracas, Venezuela. 'bspecialista en Neumonología Clínica, Doctora en Ciencias de la Salud, Coordinadora del Postgrado de Neumonología, Hospital José Ignacio Baldó, Caracas, Venezuela. cProfesor Asistente de la Cátedra de Cirugía Estomatológica, Facultad de Odontología, Universidad Central de Venezuela, Caracas, Venezuela. 'Odontóloga, Caracas, Venezuela. eProfesor Asociado de la Cátedra de Cirugía Estomatológica, Facultad de Odontología, Universidad Central de Venezuela, Caracas.

INFORMACIÓN DEL ARTÍCULO

Historia del artículo:

Recibido el 6 de enero de 2018

Aceptado el 3 de junio de 2018

Palabras clave:

Hipoxemia, EPOC, saturación de oxígeno.

\section{R E S U M E N}

Introducción: El manejo odontológico de pacientes médicamente comprometidos requiere un enfoque integral y multidisciplinario. La hipoxemia puede presentarse en pacientes odontológicos con enfermedades respiratorias durante y después de la intervención, por lo que se requiere la monitorización del porcentaje de saturación de oxígeno en sangre periférica $\left(\% \mathrm{SpO}_{2}\right)$ para identificar rápidamente aquellos trastornos que precisen intervención terapéutica. La enfermedad pulmonar obstructiva crónica (EPOC) es una enfermedad que puede permanecer subdiagnosticada en pacientes fumadores asintomáticos, quienes pueden presentar estas alteraciones.

Objetivo: Evaluar el \% $\mathrm{SpO}_{2}$ durante procedimientos de cirugía bucal en pacientes fumadores. Materiales y método: A los pacientes fumadores a quienes se realizaron procedimientos de cirugía bucal en los servicios del Postgrado de Cirugía Bucal de la Facultad de Odontología de la Universidad Central de Venezuela y aceptaron su inclusión en el estudio se les determinó el consumo tabáquico en índice paquetes/año (p/a) y se les aplicó el COPD Assesment Test (CAT), la escala de disnea modified Medical Research Council (mMRC) y la clasificación de la Global Initiative for Chronic Obstructive Lung Disease (GOLD); también se les realizó una espirometría forzada y se registró la \% $\mathrm{SpO}_{2}$ mediante un pulsioxímetro antes de comenzar el procedimiento quirúrgico, al realizar el bloqueo anestésico, un minuto después de este y al finalizar la síntesis de los tejidos. Los datos fueron analizados utilizando las pruebas t de Student, Chi cuadrado, Mann-Whitney y rho de Spearman. Se consideró un contraste estadísticamente significativo si $p<0,05$.

\footnotetext{
${ }^{*}$ Autor para correspondencia:

Correo electrónico: gissellefarinas@gmail.com (Gisselle Fariñas Fuentes).

DOI: 10.20986/recom.2019.1029/2019
}

1130-0558/@ 2019 SECOM. Publicado por Inspira Network. Este es un artículo Open Access bajo la licencia CC BY-NC-ND (http:// creativecommons.org/licenses/by-nc-nd/4.0/). 
Resultados: Se incluyeron 33 individuos, distribuidos en dos grupos: $\mathrm{G} 1, \mathrm{n}=16$, índice tabáquico $>20$ p/a; G2, $n=17$, índice tabáquico $\leq 20$ p/a. El G1 presentó mayor puntuación CAT (10 us. 4) y mMRC (2 us. 0) y una disminución en los parámetros espirométricos $\% \mathrm{VEF}_{1} / \mathrm{CVF} 59$ us. $83 \%, \mathrm{FEF}_{25-75 \%} 38$ us. $82 \mathrm{l} / \mathrm{s}$ ( $\left.p=0,001\right)$. Todos los pacientes del G1 fueron diagnosticados de EPOC y tuvieron una significante disminución en el \% $\mathrm{SpO}_{2}$ durante los tiempos quirúrgicos evaluados (97 us. 98,96 us. 98,95 us. 97,93 us. 97 \%), así como desaturaciones $\% \mathrm{SpO}_{2}<90 \%$ (75 vs. 0) y mayor porcentaje de desaturación (3,5 us. 1). No se observó correlación entre la duración de la cirugía y el \% $\mathrm{SpO}_{2}$.

Conclusiones: Los pacientes fumadores de más de 20 p/a y con diagnóstico de EPOC presentaron disminuciones significativas del $\% \mathrm{SpO}_{2}$ durante la realización de procedimientos odontológicos independientemente de su duración total; sin embargo, no se evidenciaron manifestaciones clínicas de hipoxemia.

\section{Evaluation of arterial oxygen saturation during oral surgery procedures in smoker patients}

\section{A B S T R A C T}

\section{Keywords:}

Hypoxemia, COPD, oxygen saturation.
Introduction: Hypoxemia commonly arises in dental patients with lung diseases during and after surgery and requires monitoring patients' blood oxygenation to identify life-threatening problems. Chronic Obstructive Lung Disease (COPD) could remain underdiagnosis in smokers without symptoms.

Objective: The aim of this study was to evaluate the arterial oxygen saturation $\left(\% \mathrm{SpO}_{2}\right)$ during oral surgery procedures in smoker patients.

Materials and methods: The study population consisted in thirty-three smoker patients undergoing oral surgery procedures using local anesthetic at the School of Dentistry in the Central University of Venezuela. A medical history, included information on smoking habits: pack/ year, COPD Assessment Test (CAT), modified Medical Research Council (mMRC), all patients were classified by Global Initiative for Chronic Obstructive Lung Disease criteria (GOLD). Oxygen saturation was monitored by pulse oximetry, before the beginning of the surgery, during the anesthetic administration, one minute after and the end of the suture. Data were analyzed using Student's t test, chi-squared, Mann-Whitney test, Spearman's rho test.

Results: Subjects were divided in two groups according with the smoking history: group 1 , $\mathrm{n}=16,>20$ pack/year; group 2, $\mathrm{n}=17, \leq 20$ pack/year. Group 1 have higher scores of CAT (10 vs. 4) $\mathrm{mMRC}$ (2 vs. 0) and lower spirometric values $\% \mathrm{FEV}_{1} / \mathrm{FVC} 59$ vs. $83 \%, \mathrm{FEF}_{25-75}$ of $38 \mathrm{vs} .82 \mathrm{lts} / \mathrm{s}$ $(p=0,001)$. All group 1 patients were diagnosis with COPD and lower oxygen saturation during times of surgery (97 vs. 98,96 vs. 98,95 vs. 97,93 vs. $97 \%$ ), episodes of $\% \mathrm{SpO}_{2} 90 \%$ (75 vs. $0 \%$ ) and times of desaturation below the baseline (3,5 vs. 1). There was not relation between time of surgery and oxygen saturation.

Conclusions: Patients with a smoking history $>20$ pack-years and COPD diagnosis have episodes of arterial oxygen desaturation during oral surgery procedures and it occurs in the absence of hemodynamic or other physiologic signs of hypoxemia.

\section{INTRODUCCIÓN}

El manejo odontológico de pacientes médicamente comprometidos requiere un enfoque integral y multidisciplinario. La hipoxemia puede presentarse en pacientes con enfermedades respiratorias durante y después de la intervención, por lo que se requiere la monitorización del porcentaje de saturación de oxígeno en sangre periférica $\left(\% \mathrm{SpO}_{2}\right)$ para identificar rápidamente aquellos trastornos que precisen intervención terapéutica. La utilización del oxímetro de pulso en procedimientos odontológicos permite medir el \% $\mathrm{SpO}_{2}$. Algunas investigaciones previas han reportado datos sobre la incidencia, frecuencia, gravedad y duración de la desaturación de oxígeno arterial en pacientes sometidos a procedimientos de cirugía bucal ambulatoria ${ }^{1-3}$ y otros estudios han comparado el comportamiento del $\% \mathrm{SpO}_{2}$ en diferentes intervenciones quirúrgicas, con anestesia local o sedación ${ }^{4-8}$, demostrando la importancia de la monitorización de este parámetro en la detección temprana de episodios de hipoxemia en pacientes con patologías sistémicas ${ }^{9}$. La enfermedad pulmonar obstructiva crónica (EPOC) es una entidad de alta prevalencia y comúnmente subdiagnosticada en pacientes fumadores asintomáticos, quienes pueden presentar alteraciones en el funcionalismo pulmonar ${ }^{10-13}$ e hipoxemia durante la realización de procedimientos de cirugía bucal ${ }^{14}$. No se han encontrado estudios que describan el comportamiento del $\% \mathrm{SpO}_{2}$ durante 
procedimientos odontológicos en pacientes fumadores, y en vista de que la detección temprana de episodios de hipoxemia puede prevenir complicaciones en pacientes con comorbilidades, se requiere investigar sobre el tema.

\section{OBJETIVO}

Evaluar el $\% \mathrm{SpO}_{2}$ durante los procedimientos de cirugía bucal en pacientes fumadores con y sin diagnóstico de EPOC.

\section{MATERIALES Y MÉTODO}

Estudio descriptivo, transversal, comparativo, analítico, de campo, no experimental, realizado en los Servicios de Cirugía Bucal de la Facultad de Odontología de la Universidad Central de Venezuela y Cirugía Buco-Maxilofacial del Hospital Ortopédico Infantil en Caracas-Venezuela, en pacientes a quienes se realizaron procedimientos de cirugía bucal bajo anestesia local en un periodo comprendido entre diciembre de 2015 y mayo de 2016, con una edad de entre 18 y 65 años, de cualquier sexo, fumadores y que aceptaron participar en el estudio (consentimiento informado aprobado por el Comité de Bioética de la Facultad de Odontología de la Universidad Central de Venezuela).

Fueron clasificados en dos grupos: el grupo (G1) tenía un índice tabáquico > 20 p/a y el grupo 2 (G2) un índice tabáquico de $\leq 20 \mathrm{p} / \mathrm{a}$. Cada paciente fue interrogado sobre otros factores de riesgo para la EPOC y diagnóstico previo de esta enfermedad; se aplicó el COPD Assessment Test (CAT) y la escala de disnea modified Medical Research Council (mMRC); también se les realizó una espirometría según los estándares de la American Thoracic Society. Los resultados de estas pruebas fueron evaluados por un médico especialista en Neumología, quien realizó el diagnóstico de EPOC y clasificó al paciente según la Global Initiative for Chronic Obstructive Lung Disease (GOLD) $2011^{15}$

Posteriormente a esta evaluación neumológica se llevó a cabo la cirugía. La técnica anestésica escogida fue determinada por la zona donde se realizó el procedimiento quirúrgico (infiltrativa para maxilar y troncular para mandíbula) La solución anestésica utilizada fue lidocaína más epinefrina al $2 \%$ en una concentración de 1:100.000.

Durante la realización del procedimiento quirúrgico se monitorizo el $\% \mathrm{SpO}_{2}$ mediante un pulsioxímetro marca Nonin ${ }^{\circledR}$, modelo Onyx Vantaje 9590, colocado en el dedo índice de la mano izquierda (uñas sin esmalte), permaneciendo el paciente inmóvil durante la medición. No se monitorizó la presión arterial en el brazo ipsilateral. La temperatura e iluminación del área quirúrgica se mantuvo sin variaciones durante la cirugía y no se utilizó electrocauterio.

Se registró el \% $\mathrm{SpO}_{2}$ en cuatro tiempos: 1) antes de iniciar el procedimiento, 2) durante el bloqueo anestésico, 3) un minuto después de realizar el bloqueo anestésico y 4) al finalizar la síntesis de los tejidos.

Se consideró como desaturación de oxígeno arterial un $\% \mathrm{SpO}_{2}$ menor de 90 y/o niveles menores del $2 \%$ de la medición inicial, y como desaturación clínicamente significativa una disminución mayor del 5 \%, basándonos en la investiga- ción de White y cols. ${ }^{1}$. Se clasificó según la gravedad en: leve (2\% a $5 \%$ menos de la medición basal), moderada (6 a $10 \%$ ) y grave (> $10 \%$ ). La medición de la duración del procedimiento quirúrgico se registró en minutos, desde la colocación del campo quirúrgico hasta la colocación de las gasas supralveolares.

Para el tratamiento estadístico se utilizaron estadísticas descriptivas y las siguientes pruebas: Shapiro-Wilk, chi-cuadrado, prueba U de Mann-Whitney, t de Student, prueba no paramétrica rho de Spearman y prueba no paramétrica $\mathrm{H}$ de Kruskal-Wallis. Se consideró un contraste estadísticamente significativo si $p<0,05$.

\section{RESULTADOS}

Se incluyeron en el estudio un total de 33 pacientes con edades comprendidas entre 18 y 65 años, divididos según su consumo tabáquico en G1: pacientes con índice tabáquico mayor a 20 p/a $(n=16)$ y G2: pacientes con índice tabáquico menor o igual a 20 p/a $(n=17)$, a quienes se les realizaron procedimientos de cirugía bucal: exodoncia de terceros molares, cirugías preprotésicas, biopsias y exodoncias a colgajo bajo anestesia local.

En la Tabla I se muestran las características de los grupos según indicadores clínicos y espirométricos, observándose mayor puntuación de CAT y escala de disnea en el G1. De los 33 pacientes incluidos en la investigación, se diagnosticaron 15 con EPOC: 3 de ellos (9,1 \%) notificaron al odontólogo el diagnóstico previo de la enfermedad, que fue confirmado por el médico especialista con los resultados espirométricos, CAT y mMRC, y otros 12 pacientes fueron diagnosticados en el momento de la evaluación neumológica preoperatoria. Los

\begin{tabular}{|c|c|c|c|}
\hline Variables & $\begin{array}{c}>20 \mathrm{p} / \mathrm{a} \\
\mathrm{n}=16\end{array}$ & $\begin{array}{c}<20 \mathrm{p} / \mathrm{a} \\
\mathrm{n}=17\end{array}$ & $p$ \\
\hline CAT & $10(8-14)$ & $4(2-10)$ & $0,001^{*}$ \\
\hline mMRC & & & $0,001^{*}$ \\
\hline 0 puntos & $0(0,0 \%)$ & $14(82,4 \%)$ & \\
\hline 1 punto & $6(37,5 \%)$ & $3(17,6 \%)$ & \\
\hline 2 puntos & $10(62,5 \%)$ & $0(0,0 \%)$ & \\
\hline CVF (l) & 84 & 74 & 0,037 \\
\hline $\operatorname{VEF}_{1}(\mathrm{l} / \mathrm{s})$ & 56 & 84 & $0,002^{*}$ \\
\hline $\mathrm{FEF}_{25-75 \%}(\mathrm{l} / \mathrm{s})$ & 38 & 82 & $0,001^{*}$ \\
\hline $\mathrm{VEF}_{1} / \mathrm{CVF}(\%)$ & 59 & 83 & $0,001^{*}$ \\
\hline Clasificación GOLD & & & $0,001^{*}$ \\
\hline No & $0(0 \%)$ & 17 (100\%) & \\
\hline I & $1(6,3 \%)$ & $0(0 \%)$ & \\
\hline II & $9(56,3 \%)$ & $0(0 \%)$ & \\
\hline III & $5(31,3 \%)$ & $0(0 \%)$ & \\
\hline IV & $1(6,3 \%)$ & $0(0 \%)$ & \\
\hline
\end{tabular}

*: estadísticamente significativo. CAT: COPD Assesment Test. CVF: capacidad vital forzada. $\mathrm{FEF}_{25-75 \%}$ : flujo espiratorio forzado medio. GOLD: Global Initiative for Chronic Obstructive Lung Disease. mMRC: modified Medical Research Council. p/a: paquetes/año. VEF1: volumen espiratorio forzado en el primer segundo. 
porcentajes de capacitad vital forzada (CVF), volumen espiratorio forzado en el primer segundo $\left(\mathrm{VEF}_{1}\right)$, flujo espiratorio forzado medio $\left(\mathrm{FEF}_{25-75 \%}\right.$ ) y la relación $\mathrm{VEF}_{1} / \mathrm{CVF}$ fueron menores en el G1 ( $p=0,001)$.

En la Figura $1 \mathrm{y}$ la Tabla II se muestran las variaciones del $\% \mathrm{SpO}_{2}$ durante los tiempos quirúrgicos en los grupos evaluados, evidenciándose una mayor disminución de dicho parámetro durante la cirugía en el G1 ( $p=0,001)$.

En la Tabla III se muestra que en 12 pacientes (75\%) del G1 se detectaron desaturaciones $\left(\% \mathrm{SpO}_{2}<90 \%\right)$ durante la cirugía, y este hallazgo no se observó en los pacientes del G2 $(p=0,001)$; el porcentaje de desaturación fue significativamente mayor en el G1 ( $p=0,002)$.

No se observaron diferencias estadísticamente significativas entre el \% $\mathrm{SpO}_{2}$ y la duración de la cirugía (Tabla IV).

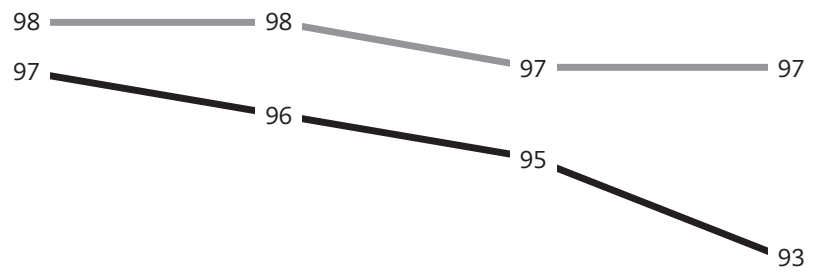

\begin{tabular}{lccc}
\hline Inicial & Anestesia & Post anestesia & Final \\
& $->20 \mathrm{paq} / \mathrm{año}$ & $-<20 \mathrm{paq} / \mathrm{año}$ &
\end{tabular}

Figura 1. Variación de la saturación arterial de oxígeno $\left(\% \mathrm{SPO}_{2}\right)$ en los diferentes tiempos quirúrgicos, según grupos.

Tabla II. Resultados de las mediciones del $\% \mathrm{SpO}_{2}$ en los diferentes tiempos quirúrgicos

\begin{tabular}{|c|c|c|c|c|c|c|c|}
\hline Paciente & Edad/Sexo & $\begin{array}{c}\text { Índice } \\
\text { tabáquico (p/a) }\end{array}$ & Procedimiento quirúrgico & $\begin{array}{l}\% \mathrm{SpO}_{2} \\
\text { inicial }\end{array}$ & $\begin{array}{c}{ }_{\% \mathrm{SpO}_{2}} \\
\text { anestesia }\end{array}$ & $\begin{array}{c}\% \mathrm{SpO}_{2} \\
\text { postanestesia }\end{array}$ & $\begin{array}{c}\% \mathrm{SpO}_{2} \\
\text { final }\end{array}$ \\
\hline 1 & $59 / F$ & 21 & Biopsia & 97 & 96 & 96 & 95 \\
\hline 2 & $46 / F$ & 40 & Cirugía preprotésica & 98 & 98 & 97 & 97 \\
\hline 3 & $50 / \mathrm{M}$ & 30 & Cirugía preprotésica & 98 & 97 & 95 & 93 \\
\hline 4 & $48 / \mathrm{M}$ & 66 & Exodoncia colgajo & 95 & 94 & 92 & 92 \\
\hline 5 & $57 / \mathrm{M}$ & 37 & Exodoncia colgajo & 98 & 96 & 95 & 93 \\
\hline 6 & $59 / \mathrm{M}$ & 45 & Exodoncia simple & 97 & 95 & 95 & 93 \\
\hline 7 & $64 / F$ & 25 & Cirugía preprotésica & 97 & 95 & 95 & 95 \\
\hline 8 & $65 / \mathrm{M}$ & 53 & Cirugía preprotésica & 98 & 96 & 93 & 93 \\
\hline 9 & $65 / \mathrm{M}$ & 50 & Cirugía preprotésica & 98 & 95 & 93 & 91 \\
\hline 10 & $64 / \mathrm{M}$ & 40 & Cirugía preprotésica & 96 & 94 & 92 & 91 \\
\hline 11 & $60 / F$ & 30 & Exodoncia colgajo & 96 & 95 & 94 & 94 \\
\hline 12 & $65 / \mathrm{M}$ & 40 & Exodoncia colgajo & 97 & 96 & 95 & 92 \\
\hline 13 & $57 / F$ & 37 & Exodoncia simple & 95 & 94 & 93 & 91 \\
\hline 14 & $47 / F$ & 21 & Exodoncia simple & 99 & 99 & 98 & 96 \\
\hline 15 & $65 / \mathrm{M}$ & 25 & Cirugía preprotésica & 98 & 97 & 95 & 94 \\
\hline 16 & $65 / F$ & 23 & Biopsia & 96 & 96 & 96 & 95 \\
\hline 17 & $28 / F$ & 3 & Exodoncia de terceros molares & 99 & 99 & 98 & 97 \\
\hline 18 & $20 / F$ & 2 & Exodoncia de terceros molares & 99 & 99 & 98 & 98 \\
\hline 19 & $29 / F$ & 3 & Exodoncia de terceros molares & 98 & 98 & 95 & 95 \\
\hline 20 & $30 / \mathrm{M}$ & 5 & Exodoncia de terceros molares & 98 & 98 & 98 & 97 \\
\hline 21 & $23 / \mathrm{M}$ & 3 & Exodoncia de terceros molares & 98 & 97 & 97 & 95 \\
\hline 22 & 19/M & 1 & Exodoncia de terceros molares & 98 & 98 & 98 & 98 \\
\hline 23 & $26 / \mathrm{M}$ & 4 & Biopsia & 99 & 98 & 97 & 97 \\
\hline 24 & $39 / \mathrm{M}$ & 10 & Cirugía preprotésica & 98 & 97 & 96 & 94 \\
\hline 25 & $40 / \mathrm{M}$ & 1 & Exodoncia colgajo & 99 & 98 & 97 & 98 \\
\hline 26 & $47 / \mathrm{M}$ & 12 & Exodoncia simple & 98 & 97 & 97 & 98 \\
\hline 27 & $38 / \mathrm{M}$ & 5 & Exodoncia simple & 98 & 97 & 97 & 98 \\
\hline 28 & $26 / \mathrm{M}$ & 1 & Exodoncia de terceros molares & 99 & 98 & 98 & 98 \\
\hline 29 & 20/M & 1 & Exodoncia de terceros molares & 99 & 98 & 97 & 97 \\
\hline 30 & $20 / \mathrm{M}$ & 2 & Exodoncia de terceros molares & 99 & 98 & 97 & 98 \\
\hline 31 & $47 / F$ & 5 & Exodoncia de terceros molares & 98 & 97 & 97 & 96 \\
\hline 32 & $18 / \mathrm{F}$ & 1 & Exodoncia de terceros molares & 98 & 98 & 97 & 96 \\
\hline 33 & $20 / \mathrm{F}$ & 3 & Exodoncia de terceros molares & 99 & 98 & 98 & 97 \\
\hline
\end{tabular}

$\% \mathrm{SpO}_{2}$ : porcentaje de saturación de oxígeno en sangre periférica. p/a: paquetes/año. 
Tabla III. Porcentaje y episodios de desaturación

$\left(\% \mathrm{SpO}_{2}<90\right)$ durante la cirugía, según grupos

\begin{tabular}{lccc} 
& $\begin{array}{c}>20 \mathrm{p} / \mathrm{a} \\
\mathrm{n}=16\end{array}$ & $\begin{array}{c}<20 \mathrm{p} / \mathrm{a} \\
\mathrm{n}=17\end{array}$ & $\mathrm{p}$ \\
\hline $\begin{array}{l}\text { Porcentaje de } \\
\text { desaturación (mediana) }\end{array}$ & $3,50 \%$ & $1,0 \%$ & 0,002 \\
$\begin{array}{l}\text { Pacientes con episodios } \\
\text { de desaturación } \\
\mathrm{n}(\%)\end{array}$ & $12(75,00 \%)$ & $0(0 \%)$ & 0,002 \\
\hline $\begin{array}{l}\% \mathrm{SpO}_{2} \text { : porcentaje de saturación de oxígeno en sangre periférica. } \\
\text { p/a: paquetes/año. }\end{array}$
\end{tabular}

\section{DISCUSIÓN}

El efecto del tabaquismo en la EPOC es bien reconocido, así como el mayor deterioro de la función pulmonar en pacientes que continúan fumando ${ }^{10-12}$. Similar a lo descrito por estos autores, encontramos que los pacientes con índice tabáquico mayor a 20 p/a tuvieron porcentajes significativamente menores de $\mathrm{VEF}_{1}, \mathrm{FEF}_{25-75 \%} \mathrm{y}$ relación $\mathrm{VEF}_{1} / \mathrm{CVF}$ en la espirometría y 15 cumplían criterios diagnósticos de la GOLD para EPOC (relación $\mathrm{VEF}_{1} / \mathrm{CVF}$ menor del 70 \%); sin embargo, solo tres pacientes refirieron haber sido diagnosticados previamente a la evaluación neumológica preoperatoria, lo que corrobora el subdiagnóstico de esta enfermedad ${ }^{13}$.

Al describir episodios de desaturación durante procedimientos odontológicos, White y cols. ${ }^{1}$ destacaron la importancia de la monitorización de este parámetro. En la presente investigación encontramos diferencias estadísticamente significativas en las variaciones del $\% \mathrm{SpO}_{2}$ durante los distintos tiempos quirúrgicos evaluados en los dos grupos, evidencián- dose una disminución más acentuada durante la cirugía en el grupo de pacientes con mayor carga tabáquica y diagnóstico de EPOC. La pulsioximetría tiene un margen de error del 1-2 \%, relacionado con: baja perfusión periférica, hemoglobinopatías, movimientos corporales, interferencia en la transmisión del haz de luz infrarrojo causada bien por esmalte de uñas, bien por la piel oscura o por ictericia, y factores ambientales ${ }^{7,16-18}$. Ningún paciente refirió antecedentes de estas patologías y el resto de las variables fueron controladas durante la realización del procedimiento. Aunque las saturaciones de un 2-3\% menos de la medición basal son difíciles de interpretar ${ }^{2}$, se ha reportado que la probabilidad de experimentar episodios de baja saturación se incrementa con la edad y es también mayor en pacientes de género masculino ${ }^{3}$; ambas características fueron observadas en este grupo de pacientes con EPOC.

Lowe y cols. ${ }^{4}$ evaluaron el $\% \mathrm{SpO}_{2}$ en pacientes que recibieron sedación con midazolam y demostraron que tuvieron mayores descensos en un $25 \%$ de estos (93-89\%). En contraste con estos hallazgos, Aeschliman y cols. ${ }^{7}$ y Shivananda y cols. ${ }^{8}$ realizaron estudios que valoraron comparativamente el $\% \mathrm{SpO}_{2}$ durante cirugía periodontal con o sin sedación oral consciente utilizando diazepam, y concluyeron que el estrés y la ansiedad fueron las causas de la alteración de la frecuencia respiratoria, la cual, en el tiempo, podría modificar los niveles de saturación de oxígeno y/o dióxido de carbono en sangre. Estas investigaciones demostraron que los pacientes premedicados con diazepam tuvieron menos desaturaciones que aquellos en los que se utilizó solo anestesia local, y que este hallazgo podría deberse a una mayor relajación durante la cirugía al ser capaces de mantener un patrón normal de respiración, a diferencia de los más ansiosos. En nuestro estudio no se utilizó sedación en ninguno de los grupos, por lo que no se puede inferir que el grupo que presentó un menor porcentaje de desaturaciones tuviera menor estrés o ansiedad que el grupo de pacientes con EPOC.

\section{Tabla IV. Duración de la cirugía y $\% \mathrm{SpO}_{2}$ en los diferentes tiempos quirúrgicos}

\begin{tabular}{|c|c|c|c|c|c|}
\hline $\begin{array}{c}\text { Duración del } \\
\text { procedimiento }\end{array}$ & & $\% \mathrm{SpO}_{2}$ inicial & $\% \mathrm{SpO}_{2}$ anestesia & $\% \mathrm{SpO}_{2}$ postanestesia & $\% \mathrm{SpO}_{2}$ final \\
\hline \multirow{3}{*}{$5-14 \mathrm{~min}$} & Mediana & 98,00 & 96,00 & 95,00 & 94,00 \\
\hline & Mínimo & 95 & 94 & 92 & 91 \\
\hline & Máximo & 99 & 99 & 98 & 98 \\
\hline \multirow{3}{*}{$15-23 \mathrm{~min}$} & Mediana & 98,00 & 97,00 & 95,00 & 95,00 \\
\hline & Mínimo & 97 & 95 & 95 & 92 \\
\hline & Máximo & 98 & 98 & 97 & 96 \\
\hline \multirow{3}{*}{ 24-32 $\min$} & Mediana & 98,00 & 98,00 & 97,00 & 97,00 \\
\hline & Mínimo & 96 & 96 & 93 & 93 \\
\hline & Máximo & 99 & 99 & 98 & 98 \\
\hline \multirow{3}{*}{$33-41 \mathrm{~min}$} & Mediana & 98,00 & 96,00 & 96,00 & 95,00 \\
\hline & Mínimo & 96 & 94 & 92 & 91 \\
\hline & Máximo & 99 & 98 & 98 & 97 \\
\hline \multirow{3}{*}{$42-50 \mathrm{~min}$} & Mediana & 98,00 & 98,00 & 97,00 & 97,00 \\
\hline & Mínimo & 98 & 97 & 95 & 93 \\
\hline & Máximo & 99 & 99 & 98 & 98 \\
\hline \multirow{3}{*}{ Total } & Mediana & 98,00 & 97,00 & 97,00 & 95,00 \\
\hline & Mínimo & 95 & 94 & 92 & 91 \\
\hline & Máximo & 99 & 99 & 98 & 98 \\
\hline
\end{tabular}

Inicial, $\mathrm{p}=0,253$. Anestesia, $\mathrm{p}=0,144$. Postanestesia, $\mathrm{p}=0,432$. Final, $\mathrm{p}=0,513$.

$\% \mathrm{SpO}_{2}$ : porcentaje de saturación de oxígeno en sangre periférica. 
La administración combinada de anestesia con vasoconstrictores puede alterar ciertos patrones hemodinámicos y se ha mencionado que la hipoxemia está solamente relacionada con pacientes sanos cuando están bajo sedación. Carrera y cols. ${ }^{6}$ demostraron, durante cirugías de terceros molares en individuos sanos, que el $\% \mathrm{SpO}_{2}$ permaneció estable en todos los tiempos de la cirugía y tanto la medida máxima en el momento de la extracción $(98,7$ \%) y el valor mínimo al finalizar la sutura (95,8 \%) se encontraron dentro de los valores normales (hipoxemia definida como $\mathrm{SpO}_{2}<95 \%$ ), a pesar de que consideraron que todos estos pacientes estarían en riesgo de sufrir hipoxemia, principalmente debido a la tendencia de estos de hacer apnea en los momentos de gran ansiedad. En contraste con estos resultados, en nuestro estudio, que incluyó solo fumadores, en un $75 \%$ de los pacientes del grupo con alta carga tabáquica se detectaron desaturaciones $\left(\mathrm{SpO}_{2}<90 \%\right)$ y el porcentaje de desaturación también fue significantemente mayor en este grupo.

Matthews y cols. ${ }^{5}$ y Gandy y cols. ${ }^{9}$ describieron episodios de hipoxemia al final de la cirugía, atribuyendo estos hallazgos al aumento de la demanda de oxígeno en los tejidos debido al incremento de catecolaminas en el torrente sanguíneo secundario al estrés acumulado. En concordancia con estos hallazgos, encontramos que los registros del \% $\mathrm{SpO}_{2}$ al final de la cirugía oscilaron entre el $91 \%$ y el $97 \%$, con una mediana del $93 \%$ en el grupo de fumadores con EPOC; sin embargo, en el grupo de fumadores $\leq 20$ paquetes/año solo se detectó en un paciente un $\% \mathrm{SpO}_{2}$ de 94 (mediana de $97 \%$ ), diferencias estadísticamente significativas. La investigación de Matthews y cols. ${ }^{5}$, que incluyó 18 pacientes fumadores, contrasta con nuestros resultados, ya que ellos no observaron diferencias significativas en los valores iniciales del $\% \mathrm{SpO}_{2}$, al igual que en el resto de los tiempos quirúrgicos.

Hovagim y cols. ${ }^{2}$ demostraron una fuerte correlación entre la incidencia de desaturaciones y la alta carga tabáquica, similar a lo observado en esta investigación, y aunque no se encontraron estudios en la bibliografía revisada que incluyeran evaluaciones del $\% \mathrm{SpO}_{2}$ durante procedimientos odontológicos en pacientes con EPOC para realizar comparaciones con nuestros resultados, hay que resaltar que se evidenciaron desaturaciones en ambos grupos (alta o baja carga tabáquica), pero significativamente mayores en el grupo de pacientes con EPOC. Se afirma que la relación de transformación anormal de ventilación/perfusión (V/Q) en las diversas regiones del pulmón es la causa más común de hipoxemia en todos los tipos de pacientes, incluyendo los que tienen $\mathrm{EPOC}$, quienes también pueden presentar hipoventilación; la mayoría de estos enfermos tienen una gasometría arterial normal o poco alterada durante largo tiempo, y la hipoxemia aparece en etapas avanzadas de la enfermedad ${ }^{14}$.

Cortos episodios de hipoxemia en pacientes sanos pueden tener escasas consecuencias, pero en pacientes con EPOC la detección temprana podría evitar complicaciones mayores. La hipoxemia aguda con un $\% \mathrm{SpO}_{2}$ menor de 80 puede sospecharse por la presencia de cianosis; sin embargo, puede manifestarse a través de signos inespecíficos, como taquicardia, taquipnea y alteraciones del sistema nervioso central ${ }^{14}$.

La principal limitación de nuestro estudio fue el pequeño número de la muestra y que no se monitorizaron ni la frecuencia cardiaca ni la respiratoria durante toda la cirugía, lo que podría ser objeto de futuras investigaciones. Sin embargo, ningún paciente presentó un $\% \mathrm{SpO}_{2}$ menor de 80 y, por tanto, no se detectaron manifestaciones neurológicas ni cianosis durante la realización de los procedimientos.

Aeschliman y cols. ${ }^{7}$ no encontraron en su estudio relación entre la duración de los procedimientos y el tratamiento realizado, con respecto al \% $\mathrm{SpO}_{2}$, hallazgos que concuerdan con los nuestros, donde tampoco evidenciamos diferencias estadísticamente significativas entre estas variables.

\section{CONCLUSIONES}

Los resultados de esta investigación nos permiten concluir que existe un número importante de pacientes fumadores asintomáticos atendidos en las consultas odontológicas que pueden tener EPOC y desconocerlo, por lo que podría ser esta una oportunidad de intervención del odontólogo en el momento de realizar la historia clínica, que podría remitir a consultas médicas especializadas a los pacientes con índice tabáquico mayor de 20 p/a.

La pulsioximetría permitió detectar variaciones del $\% \mathrm{SpO}_{2}$ durante los distintos tiempos quirúrgicos en los dos grupos evaluados, evidenciándose una disminución más acentuada en el grupo de pacientes con EPOC.

Aunque en las dos terceras partes del grupo de pacientes con mayor carga tabáquica se detectaron desaturaciones $\left(\% \mathrm{SpO}_{2}<90 \%\right)$ y el porcentaje de desaturación también fue significativamente mayor, no se registraron manifestaciones clínicas relevantes, lo que confirma la seguridad de los procedimientos odontológicos bajo anestesia local. Sin embargo, no deja de ser importante la monitorización de este parámetro en pacientes con tabaquismo.

\section{CONFLICTO DE INTERESES}

Los autores afirman que no existe conflicto de intereses en la realización de esta investigación.

\section{B I B L I O G R A F Í A}

1. White C, Dolwick F, Gravenstein N, Paulus D. Incidence of oxygen desaturation during oral surgery outpatient procedures. J Oral Maxillofac Surg. 1989;47(2):147-9. DOI: 10.1016/s02782391(89)80105-3.

2. Hovagim A, Vitkun S, Manecke G, Reiner R. Arterial oxygen desaturation in adult dental patients receiving conscious sedation. J Oral Maxillofac Surg. 1989;47(9):936-9. DOI: 10.1016/02782391(89)90377-7.

3. Viljoen A, Byth K, Coombs M, Mahoney G, Stewart D. Dental intravenous sedations: A retrospective quality assurance of 3500 cases. Anesth Prog. 2011;58(3):113-20. DOI: 10.2344/09-00001.1.

4. Lowe T, Brook I. Oxygen saturation during third molar removal with local anaesthetic alone and in combination with intravenous sedation. Br Dent J. 1991;171(7):210-3. DOI: 10.1038/sj.bdj.4807662.

5. Matthews R, Malkawi Z, Griffiths M, Scully C. Pulse oximetry during minor oral surgery with and without intravenous sedation. Oral Surg Oral Med Oral Pathol. 1992;74(5):537-43. DOI: 10.1016/0030-4220(92)90339-r.

6. Carrera I, Mestre R, Berini L, Gay-Escoda C. Alterations in monitored vital constants induced by various local anesthetics in 
combination with different vasoconstrictors in the surgical removal of lower third molars. Bull Group Int Rech Sci Stomatol Odontol. 2000;42(1):1-10.

7. Aeschliman S, Blue M, Williams K, Cobb C, McNeill S. A preliminary study on oxygen saturation levels of patients during periodontal surgery with and without oral conscious sedation using diazepam. 2003;4(7):1056-9. DOI: 10.1902/jop.2003.74.7.1056.

8. Shivananda H, Raghava K, Sudhakar S, Thomas B, Dayakar M. Comparative evaluation of oxygen saturation during periodontal surgery with or without oral conscious sedation in anxious patients. J Indian Soc Periodontol. 2014;18(6):718-22. DOI: 10.4103/0972-124X.147406.

9. Gandy SR. The use of pulse oximetry in dentistry. J Am Dent Assoc. 1995;126(9):1274-8. DOI: 10.14219/jada.archive.1995.0363.

10. Fletcher C, Peto R. The natural history of chronic airflow obstruction. BMJ. 1977;1(6077):1645-48. DOI: 10.1136/bmj.1.6077.1645.

11. Anthonisen N, Connett J, Kiley J, Altose M, Bailey W, Buist A. Effects of smoking intervention and the use of an inhaled anticholinergic bronchodilator on the rate of decline of FEV1. The Lung Health Study. J Am Med Assoc. 1994;272(19): 1497-505.
12. Celli B, Thomas N, Anderson J, Ferguson G, Jenkins C, Jones P. Effect of pharmacotherapy on rate of decline of lung function in chronic obstructive pulmonary disease: results from the TORCH study. Am J Respir Crit Care Med. 2008;178(4):332-8. DOI: 10.1164/rccm.200712-18690C.

13. Talamo C, De Oca M, Halbert R, Pérez-Padilla R, Jardim J, Muiño A. Diagnostic labeling of COPD in five Latin American cities. Chest. 2007;131(1):60-7. DOI: 10.1378/chest.06-1149.

14. Singh V, Gupta P, Khatana S, Bhagol A. Supplemental oxygen therapy: Important considerations in oral and maxillofacial surgery. Natl J Maxillofac Surg. 2011;2(1);10-4. DOI: 10.4103/0975-5950.85846.

15. Global Strategy for the Diagnosis, Management, and Prevention of Chronic Obstructive Pulmonary Disease. Update 2011 [citado 2012 Feb 1]. Disponible en: www.goldcopd.org

16. Schnapp LM, Cohen NH. Pulse oximetry: uses and abuses. Chest. 1990;98(5):1244-50. DOI: 10.1378/chest.98.5.1244.

17. Council on Scientific Affairs, American Medical Association. The use of pulse oximetry during conscious sedation. JAMA. 1993;270(12):1463-8.

18. Mardirossian G, Schneider RE. Limitations of pulse oximetry. Anesth Prog. 1992;39:194-6. 